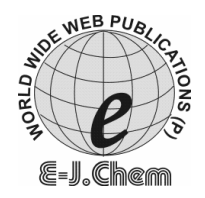

http://www.e-journals.net
ISSN: 0973-4945; CODEN ECJHAO

E-Journal of Chemistry 2010, 7(3), 927-934

\title{
Evaluation of Excess Thermodynamic Parameters in a Binary Liquid Mixture $($ Cyclohexane $+O$-Xylene) at Different Temperatures
}

\author{
K NARENDRA*, P. NARAYANAMURTHY ${ }^{\S}$ and CH. SRINIVASU \\ *Department of Physics, \\ V.R.Siddhartha Engg.College, Vijayawada, Andhrapradesh- 520007, India. \\ ${ }^{\S}$ Department of Physics, Acharya Nagarjuna University, Nagarjuna nagar, Guntur, India. \\ Department of Physics, Andhra Loyala College, Vijayawada, India. \\ kollanarendra@rediff.com
}

Received 24 December 2009; Accepted 20 February 2010

\begin{abstract}
The ultrasonic velocity, density and viscosity in binary liquid mixture cyclohexane with $o$-xylene have been determined at different temperatures from 303.15 to $318.15 \mathrm{~K}$ over the whole composition range. The data have been utilized to estimate the excess adiabatic compressibility $\left(\beta^{\mathrm{E}}\right)$, excess volumes $\left(\mathrm{V}^{\mathrm{E}}\right)$, excess intermolecular free length $\left(\mathrm{L}_{\mathrm{f}}^{\mathrm{E}}\right)$, excess internal pressure $\left(\pi^{\mathrm{E}}\right)$ and excess enthalpy $\left(\mathrm{H}^{\mathrm{E}}\right)$ at the above temperatures. The excess values have been found to be useful in estimating the strength of the interactions in the liquid mixtures. Analysis of these parameters indicates that there are weak interactions among the components of the binary mixtures.
\end{abstract}

Keywords: Ultrasonic velocity, Binary mixtures, Excess thermodynamic parameters.

\section{Introduction}

Ultrasonic study of liquid and liquid mixtures has gained much importance during the last two decades in assessing the nature of molecular interactions and investigating the physicochemical behaviour of such systems. A survey of literature ${ }^{1-4}$ indicates that excess values of ultrasonic velocity, adiabatic compressibility and molar volume in liquid mixtures are useful in understanding the interactions between the molecules. The adiabatic compressibility deviations are more related with structural effects and packing phenomena. The systematic investigations of these excess properties are therefore of great importance. The present investigation is a study of temperature variation of $u, \rho, \eta, \beta$, molar volume (V), 
intermolecular free length $\left(\mathrm{L}_{\mathrm{f}}\right)$, excess adiabatic compressibility $\left(\beta^{\mathrm{E}}\right)$, excess molar volume $\left(\mathrm{V}^{\mathrm{E}}\right)$, excess intermolecular free length $\left(\mathrm{L}_{\mathrm{f}}^{\mathrm{E}}\right)$, excess internal pressure $\left(\pi^{\mathrm{E}}\right)$ and excess enthalpy $\left(\mathrm{H}^{\mathrm{E}}\right)$ in cyclohexane $+o$-xylene.

\section{Experimental}

The chemicals were redistilled and purified by the standard methods described ${ }^{5,6}$. Liquid mixtures of different known compositions were prepared by mixing measured amounts of the pure liquids in cleaned and dried flasks. Ultrasonic velocity was measured by a single crystal variable path interferometer (Mittal, Model F-81) at a frequency of $3 \mathrm{MHz}$. The accuracy of the velocity measurements is $\pm 5 \mathrm{~ms}^{-1}$. The densities of pure liquids and liquid mixtures were measured by employing a specific gravity bottle at all the temperatures and weights were taken to an accuracy of $\pm 0.1 \mathrm{mg}$.

The Viscosities were measured with Ostwald viscometer. The viscometer was calibrated at each temperature using redistilled water. The measurements were made at all the temperatures with the help of thermostat with an accuracy of $\pm 0.1 \mathrm{~K}$. Acoustical parameters such as adiabatic compressibility $(\beta)$, intermolecular free length $\left(L_{f}\right)$, molar volume $(\mathrm{Vm})$, Enthalpy $(\mathrm{H})$ and internal pressure $(\pi)$ were calculated using standard equations.

\section{Theory}

The adiabatic compressibility has been determined by using the experimentally measured ultrasonic velocity $(\mathrm{U})$ and density $(\rho)$ by the following formula

$$
\beta_{a d}=\frac{1}{\rho U^{2}}
$$

The molar volumes of the binary mixtures were calculated using the equation

$$
\mathrm{V}=\left(\mathrm{X}_{1} \mathrm{M}_{1}+\mathrm{X}_{2} \mathrm{M}_{2}\right) / \rho
$$

Inter Molecular free length $\left(\mathrm{L}_{\mathrm{f}}\right)$ was calculated by using the relation

$$
\mathrm{L}_{\mathrm{f}}=\mathrm{K}\left(\beta_{\mathrm{ad}}\right)^{1 / 2}
$$

Where, $\mathrm{K}$ is temperature dependent Jacobson's constant ${ }^{2}$. Enthalpy $(\mathrm{H})$ is calculated using the relation

$$
\mathrm{H}=\Pi . \mathrm{V}_{\mathrm{m}}
$$

Internal pressure $(\Pi)$ is calculated using the formula

$$
I I=b R T{\frac{(K h)^{1 / 2}}{U}}^{2 / 3} \cdot \eta^{7 / 6}
$$

The excess properties such as $\beta^{\mathrm{E}}, \mathrm{Vm}^{\mathrm{E}} \mathrm{L}_{\mathrm{f}}^{\mathrm{E}}, \mathrm{H}^{\mathrm{E}}$ and $\pi^{\mathrm{E}}$ have been calculated using the equation

$$
\mathrm{Y}^{\mathrm{E}}=\mathrm{Y}_{\text {mix }}-\left[\mathrm{X}_{1} \mathrm{Y}_{1}+\mathrm{X}_{2} \mathrm{Y}_{2}\right]
$$

Where $\mathrm{Y}^{\mathrm{E}}$ is $\beta^{\mathrm{E}}$ or $\mathrm{Vm}^{\mathrm{E}}$ or $\mathrm{L}_{\mathrm{f}}^{\mathrm{E}}$ or $\mathrm{H}^{\mathrm{E}}$ or $\pi^{\mathrm{E}}$, and $\mathrm{X}$ represents mole fraction of the component and subscripts 1 and 2 stand for the components 1 and 2 .

\section{Results and Discussion}

The values of $u, \rho, \eta, \beta, V$ and $L_{f}$ for the binary system at different temperatures are given in Table 1. 
Table 1. Ultrasonic velocity (u), density $(\rho)$ and viscosity $(\eta)$, adiabatic compressibility $(\beta)$, molar volume $(\mathrm{Vm})$, intermolecular free length $\left(\mathrm{L}_{\mathrm{f}}\right)$ values for cylohexane with $o$-xylene.

\begin{tabular}{|c|c|c|c|c|c|c|}
\hline \multicolumn{7}{|c|}{ Temperature at $303.15 \mathrm{~K}$} \\
\hline $\mathrm{X}_{1}$ & $\mathrm{u} \mathrm{ms}^{-1}$ & $\begin{array}{c}\rho \\
\mathrm{Kg} \mathrm{m}^{-3}\end{array}$ & $\begin{array}{c}\eta \\
/ 10^{-3} \mathrm{Nsm}^{-2}\end{array}$ & $\begin{array}{l}\beta \times 10^{12} \\
\mathrm{~m}^{2} \mathrm{~N}^{-1}\end{array}$ & $\begin{array}{c}\mathrm{Vm} \\
\mathrm{cm}^{3} \mathrm{~mol}^{-1}\end{array}$ & $\mathrm{~L}_{\mathrm{f}} \mathrm{A}^{\mathrm{o}}$ \\
\hline 0.0000 & 1235.6 & 0.7678 & 0.8224 & 85.3058 & 109.6119 & 0.5791 \\
\hline 0.0908 & 1237.5 & 0.7768 & 0.7406 & 84.0621 & 110.9140 & 0.5749 \\
\hline 0.1835 & 1243.6 & 0.7865 & 0.6960 & 82.2063 & 112.1388 & 0.5685 \\
\hline 0.2781 & 1250.6 & 0.7975 & 0.6700 & 80.1706 & 113.2023 & 0.5614 \\
\hline 0.3747 & 1259.0 & 0.8096 & 0.6540 & 77.9252 & 114.1358 & 0.5535 \\
\hline 0.4734 & 1267.5 & 0.8222 & 0.6504 & 75.7054 & 115.0268 & 0.5455 \\
\hline 0.5742 & 1274.7 & 0.8304 & 0.6558 & 74.1076 & 116.5612 & 0.5398 \\
\hline 0.6772 & 1284.9 & 0.8368 & 0.6678 & 72.3779 & 118.3771 & 0.5334 \\
\hline 0.7824 & 1300.5 & 0.8486 & 0.6820 & 69.6749 & 119.4594 & 0.5234 \\
\hline 0.8900 & 1316.2 & 0.8614 & 0.6951 & 67.0067 & 120.4319 & 0.5132 \\
\hline 1.0000 & 1338.7 & 0.8707 & 0.7051 & 64.0815 & 121.9249 & 0.5019 \\
\hline \multicolumn{7}{|c|}{ at $308.15 \mathrm{~K}$} \\
\hline 0.0000 & 1206.1 & 0.7625 & 0.7539 & 90.1520 & 110.3738 & 0.5996 \\
\hline 0.0908 & 1210.2 & 0.7731 & 0.6895 & 88.3109 & 111.4448 & 0.5934 \\
\hline 0.1835 & 1218.7 & 0.7831 & 0.6525 & 85.9713 & 112.6257 & 0.5855 \\
\hline 0.2781 & 1227.5 & 0.7946 & 0.6301 & 83.5235 & 113.6155 & 0.5771 \\
\hline 0.3747 & 1237.3 & 0.8070 & 0.6135 & 80.9327 & 114.5035 & 0.5681 \\
\hline 0.4734 & 1245.0 & 0.8194 & 0.6093 & 78.7345 & 115.4199 & 0.5603 \\
\hline 0.5742 & 1254.2 & 0.8280 & 0.6135 & 76.7718 & 116.8990 & 0.5533 \\
\hline 0.6772 & 1262.8 & 0.8349 & 0.6216 & 75.1008 & 118.6465 & 0.5473 \\
\hline 0.7824 & 1278.6 & 0.8466 & 0.6327 & 72.2496 & 119.7416 & 0.5368 \\
\hline 0.8900 & 1294.7 & 0.8600 & 0.6486 & 69.3633 & 120.6280 & 0.5259 \\
\hline 1.0000 & 1315.0 & 0.8694 & 0.6652 & 66.5164 & 122.1072 & 0.5150 \\
\hline \multicolumn{7}{|c|}{ at $313.15 \mathrm{~K}$} \\
\hline 0.0000 & 1188.7 & 0.7587 & 0.7042 & 93.2715 & 110.9266 & 0.6142 \\
\hline 0.0908 & 1192.6 & 0.7711 & 0.6314 & 91.1762 & 111.7339 & 0.6073 \\
\hline 0.1835 & 1201.8 & 0.7811 & 0.5997 & 88.6288 & 112.9141 & 0.5987 \\
\hline 0.2781 & 1209.3 & 0.7930 & 0.5801 & 86.2194 & 113.8447 & 0.5906 \\
\hline 0.3747 & 1219.5 & 0.8054 & 0.5718 & 83.4881 & 114.7310 & 0.5811 \\
\hline 0.4734 & 1228.7 & 0.8181 & 0.5684 & 80.9494 & 115.5891 & 0.5722 \\
\hline 0.5742 & 1237.6 & 0.8270 & 0.5701 & 78.9435 & 117.0404 & 0.5651 \\
\hline 0.6772 & 1245.5 & 0.8337 & 0.5798 & 77.3219 & 118.8173 & 0.5593 \\
\hline 0.7824 & 1261.8 & 0.8453 & 0.5925 & 74.2945 & 119.8258 & 0.5482 \\
\hline 0.8900 & 1278.7 & 0.8585 & 0.6055 & 71.2342 & 120.8387 & 0.5368 \\
\hline 1.0000 & 1297.5 & 0.8677 & 0.6211 & 68.4567 & 122.3464 & 0.5262 \\
\hline \multicolumn{7}{|c|}{ at $318.15 \mathrm{~K}$} \\
\hline 0.0000 & 1168.1 & 0.7531 & 0.6562 & 97.3125 & 111.7514 & 0.6318 \\
\hline 0.0908 & 1171.8 & 0.7668 & 0.5798 & 94.8148 & 112.3605 & 0.6242 \\
\hline 0.1835 & 1181.1 & 0.7787 & 0.5491 & 92.0530 & 113.2621 & 0.6145 \\
\hline 0.2781 & 1188.7 & 0.7907 & 0.5328 & 89.4967 & 114.1759 & 0.6059 \\
\hline 0.3747 & 1198.0 & 0.8034 & 0.5233 & 86.7270 & 115.0166 & 0.5965 \\
\hline
\end{tabular}




\begin{tabular}{lllllll}
\hline 0.4734 & 1207.5 & 0.8158 & 0.5195 & 84.0702 & 115.9292 & 0.5873 \\
0.5742 & 1216.0 & 0.8254 & 0.5209 & 81.9252 & 117.2672 & 0.5797 \\
0.6772 & 1224.3 & 0.8329 & 0.5259 & 80.0900 & 118.9314 & 0.5732 \\
0.7824 & 1240.1 & 0.8440 & 0.5355 & 77.0418 & 120.1105 & 0.5622 \\
0.8900 & 1259.7 & 0.8567 & 0.5496 & 73.5534 & 121.0926 & 0.5493 \\
1.0000 & 1278.7 & 0.8659 & 0.5691 & 70.6254 & 122.6008 & 0.5383 \\
\hline
\end{tabular}

The plots of ultrasonic velocity against mole fraction of $o$-xylene at different temperatures are shown in Figure 1. These plots are almost linear with either positive or negative slope indicating the existence of very weak intermolecular attractions in these systems and there is a little deviation from ideal behavior. These forces may be of induced dipole induced dipole type.

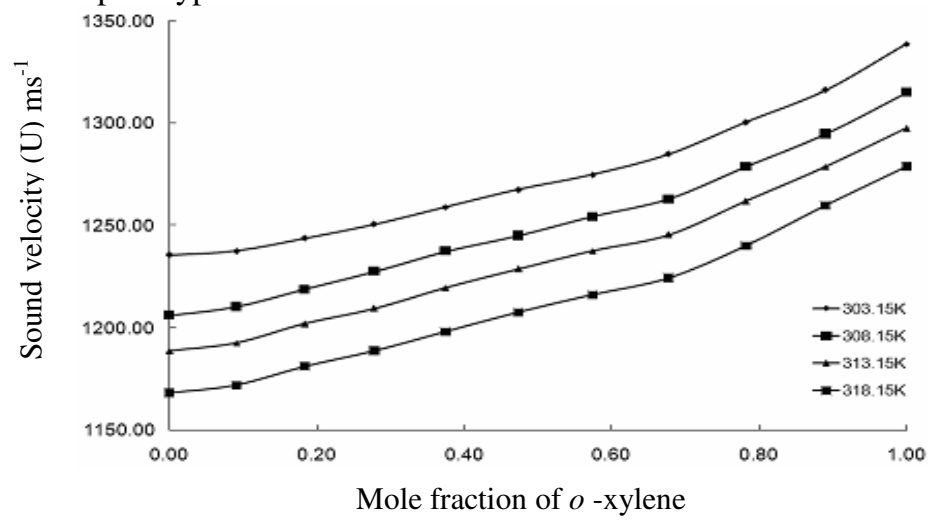

Figure 1. Plots of ultrasonic velocity versus mole fraction of $o$-xylene with cyclohexane at different temperatures.

Excess values of adiabatic compressibility $\left(\beta^{\mathrm{E}}\right)$, molar volume $\left(\mathrm{Vm}^{\mathrm{E}}\right)$, intermolecular free length $\left(\mathrm{L}_{\mathrm{f}}^{\mathrm{E}}\right)$, Enthalpy $\left(\mathrm{H}^{\mathrm{E}}\right)$ and internal pressure $\left(\pi^{\mathrm{E}}\right)$ are calculated for the system investigated are presented in Table 2.

Table 2. Excess values of adiabatic compressibility $\left(\beta^{\mathrm{E}}\right)$, molar volume $\left(\mathrm{Vm}^{\mathrm{E}}\right)$, intermolecular free length $\left(\mathrm{L}_{\mathrm{f}}^{\mathrm{E}}\right)$, enthalpy $\left(\mathrm{H}^{\mathrm{E}}\right)$ and internal pressure $\left(\pi^{\mathrm{E}}\right)$ for cylohexane with $o$-xylene.

\begin{tabular}{cccccc}
\hline \multicolumn{7}{c}{ Temperature at $303.15 \mathrm{~K}$} \\
\hline $\mathrm{X}_{1}$ & $\beta^{\mathrm{E}}$ & $\mathrm{Vm}^{\mathrm{E}}$ & $\mathrm{L}_{\mathrm{f}}^{\mathrm{E}}$ & $\mathrm{H}^{\mathrm{E}}$ & $\pi^{\mathrm{E}}$ \\
\hline 0.0000 & 0.0000 & 0.0000 & -0.0037 & 0.00 & 0.00 \\
0.0908 & 0.6839 & 0.1839 & -0.0009 & -19009.59 & -187.68 \\
0.1835 & 0.7953 & 0.2674 & -0.0001 & -28635.22 & -282.02 \\
0.2781 & 0.7679 & 0.1658 & 0.0002 & -33079.01 & -320.90 \\
0.3747 & 0.5731 & -0.0903 & -0.0002 & -34726.08 & -328.20 \\
0.4734 & 0.4474 & -0.4142 & -0.0005 & -32571.97 & -298.73 \\
0.5742 & 0.9888 & -0.1209 & 0.0016 & -26742.32 & -253.29 \\
0.6772 & 1.4447 & 0.4271 & 0.0032 & -19040.03 & -197.00 \\
0.7824 & 0.9754 & 0.2136 & 0.0014 & -12147.85 & -126.08 \\
0.8900 & 0.5906 & -0.1386 & -0.0004 & -5552.76 & -3.16 \\
1.0000 & 0.0000 & 0.0000 & -0.0032 & 0.00 & 0.00 \\
\hline \multicolumn{7}{c}{ at 308.15 K } \\
\hline 0.0000 & 0.0000 & 0.0000 & -0.0047 & 0.00 & 0.00 \\
\hline \multicolumn{7}{c}{}
\end{tabular}




\begin{tabular}{|c|c|c|c|c|c|}
\hline 0.0908 & 0.2950 & -0.0019 & -0.0031 & -16592.75 & -157.48 \\
\hline 0.1835 & 0.1378 & 0.0857 & -0.0031 & -25615.40 & -245.89 \\
\hline 0.2781 & -0.0800 & -0.0388 & -0.0035 & -30224.66 & -285.47 \\
\hline 0.3747 & -0.3916 & -0.2867 & -0.0043 & -33057.25 & -303.70 \\
\hline 0.4734 & -0.2595 & -0.5287 & -0.0036 & -31428.22 & -281.62 \\
\hline 0.5742 & 0.1605 & -0.2314 & -0.0021 & -26663.17 & -245.92 \\
\hline 0.6772 & 0.9268 & 0.3100 & 0.0007 & -20195.90 & -200.48 \\
\hline 0.7824 & 0.5691 & 0.1744 & -0.0009 & -14404.02 & -141.65 \\
\hline 0.8900 & 0.2347 & -0.1958 & -0.0025 & -7361.82 & -65.56 \\
\hline 1.0000 & 0.0000 & 0.0000 & -0.0041 & 0.00 & 0.00 \\
\hline \multicolumn{6}{|c|}{ at $313.15 \mathrm{~K}$} \\
\hline 0.0000 & 0.0000 & 0.0000 & -0.0058 & 0.00 & 0.00 \\
\hline 0.0908 & 0.1474 & -0.2373 & -0.0046 & -20734.84 & -185.70 \\
\hline 0.1835 & -0.1088 & -0.1215 & -0.0050 & -28883.09 & -266.63 \\
\hline 0.2781 & -0.1769 & -0.2755 & -0.0047 & -32851.84 & -299.51 \\
\hline 0.3747 & -0.5151 & -0.4949 & -0.0056 & -33297.45 & -297.82 \\
\hline 0.4734 & -0.6075 & -0.7643 & -0.0057 & -31765.24 & -276.19 \\
\hline 0.5742 & -0.1117 & -0.4632 & -0.0039 & -27672.53 & -246.63 \\
\hline 0.6772 & 0.8256 & 0.1400 & -0.0006 & -20391.32 & -196.13 \\
\hline 0.7824 & 0.4161 & 0.0508 & -0.0023 & -13971.94 & -133.80 \\
\hline 0.8900 & 0.0349 & -0.2589 & -0.0042 & -7595.69 & -65.45 \\
\hline 1.0000 & 0.0000 & 0.0000 & -0.0050 & 0.00 & 0.00 \\
\hline \multicolumn{6}{|c|}{ at $318.15 \mathrm{~K}$} \\
\hline 0.0000 & 0.0000 & 0.0000 & -0.0064 & 0.00 & 0.00 \\
\hline 0.0908 & -0.0857 & -0.5596 & -0.0055 & -23578.41 & -199.22 \\
\hline 0.1835 & -0.3834 & -0.4941 & -0.0063 & -31857.42 & -279.35 \\
\hline 0.2781 & -0.4218 & -0.6112 & -0.0060 & -34774.78 & -304.18 \\
\hline 0.3747 & -0.6179 & -0.8212 & -0.0063 & -35350.15 & -304.23 \\
\hline 0.4734 & -0.6438 & -0.9798 & -0.0062 & -33607.99 & -284.25 \\
\hline 0.5742 & -0.0984 & -0.7345 & -0.0042 & -29243.33 & -251.08 \\
\hline 0.6772 & 0.8183 & -0.1852 & -0.0010 & -23087.77 & -208.85 \\
\hline 0.7824 & 0.5857 & -0.1435 & -0.0021 & -16775.10 & -151.16 \\
\hline 0.8900 & -0.0215 & -0.3225 & -0.0048 & -9581.94 & -79.85 \\
\hline 1.0000 & 0.0000 & 0.0000 & -0.0054 & 0.00 & 0.00 \\
\hline
\end{tabular}

The variation of $\beta^{\mathrm{E}}$ with mole fraction of $o$-xylene at different temperatures of study is shown in Figure 2.

Figure 2 shows the variation of excess adiabatic compressibility with mole fraction of $o$-xylene. $\beta^{\mathrm{E}}$ shows negative magnitude at lower mole fraction at all temperatures except $303.15 \mathrm{~K}$. However, it becomes positive and of increase nature with increase in mole fraction of $o$-xylene. The positive excess compressibility suggests the medium is weakly packed. In the lower concentration of $o$-xylene $\beta^{\mathrm{E}}$ is negative it suggests the medium is highly packed. It may also because of spherical in shape of cyclohexane. Hence at higher mol fraction of $o$-xylene the packing environment is lost and the system may be having weak interaction. The same is reflected in variation in excess free length Figure 3.

The variation of $\mathrm{V}_{\mathrm{m}}^{\mathrm{E}}$ with mole fraction of $o$-xylene at different temperatures of study is shown in Figure.4. 


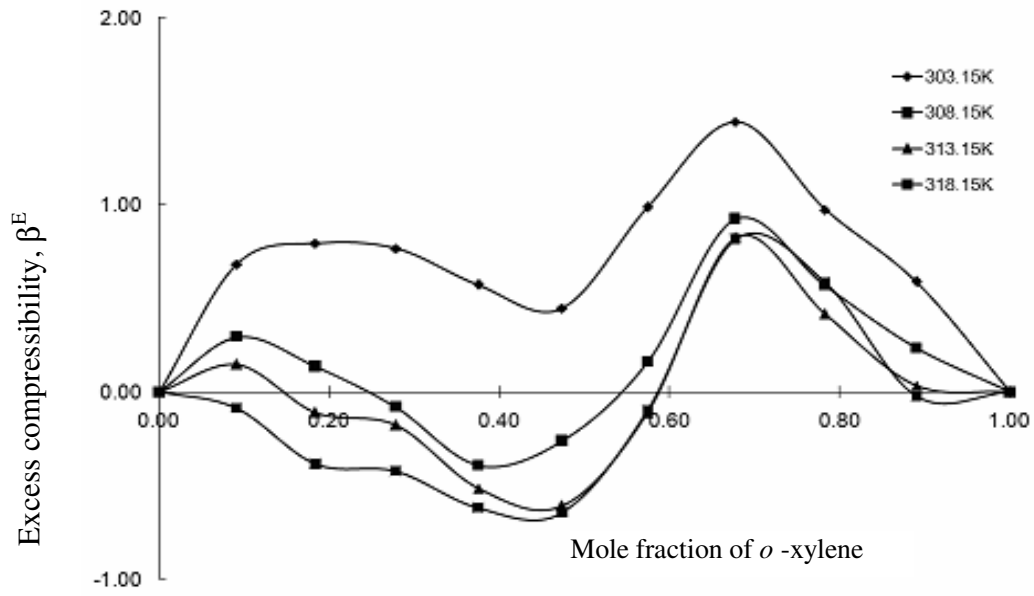

Figure 2. Variation of excess compressibility with mole fraction at different temperatures.

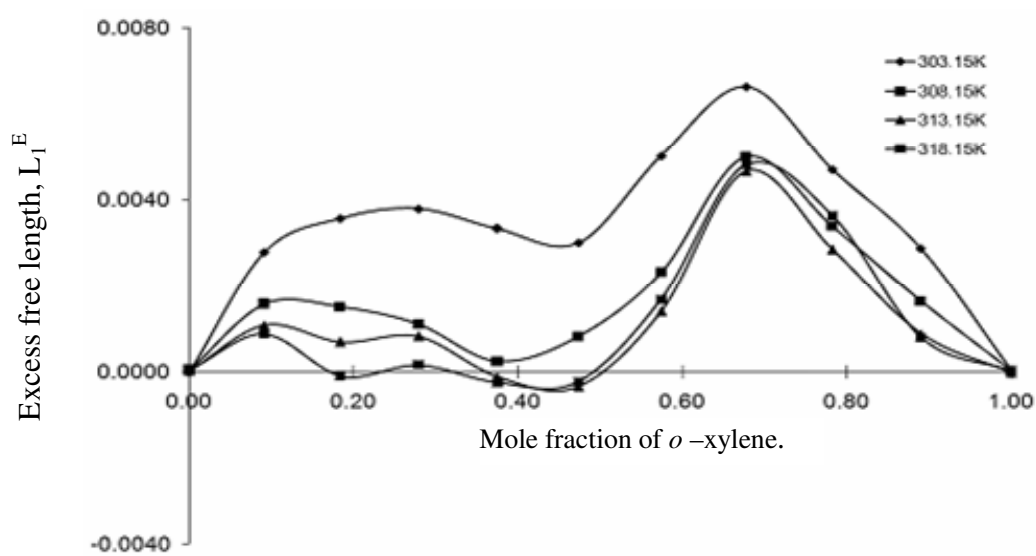

Figure 3. Variation of excess free length with mole fraction at different temperatures.

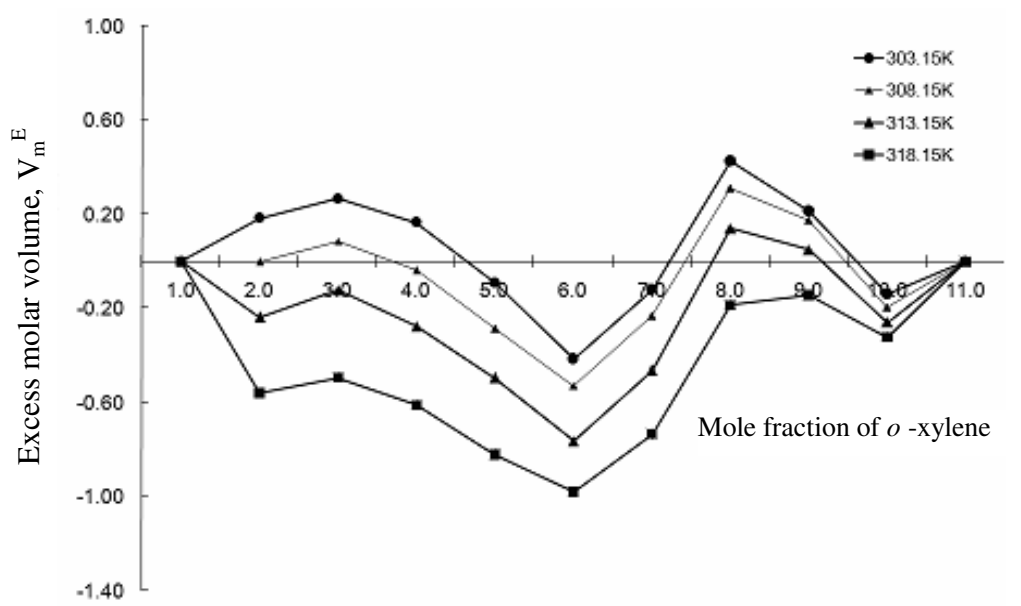

Figure 4. Variation of excess molar volume with mole fraction at different temperatures. 
It is observed that the values of $\mathrm{V}_{\mathrm{m}}{ }^{\mathrm{E}}$ are negative at higher temperatures. The values are less at all the temperatures and minimum at $x_{1}=0.5742$ and maximum at $x_{1}=0.7824$. The negative values can be visualized as being due to a closer approach of the unlike molecules in the liquid mixtures, this indicating the existence of specific interactions between the liquid components. Since liquids having significantly different molecular sizes are considered, the $\mathrm{V}_{\mathrm{m}}{ }^{\mathrm{E}}$ values show positive deviations at some places and negative at others. This signifies weak interactions.

The variation of $\mathrm{H}^{\mathrm{E}}, \pi^{\mathrm{E}}$ with mole fraction of $o$-xylene at different temperatures of study is shown in Figure 5 \& 6 .

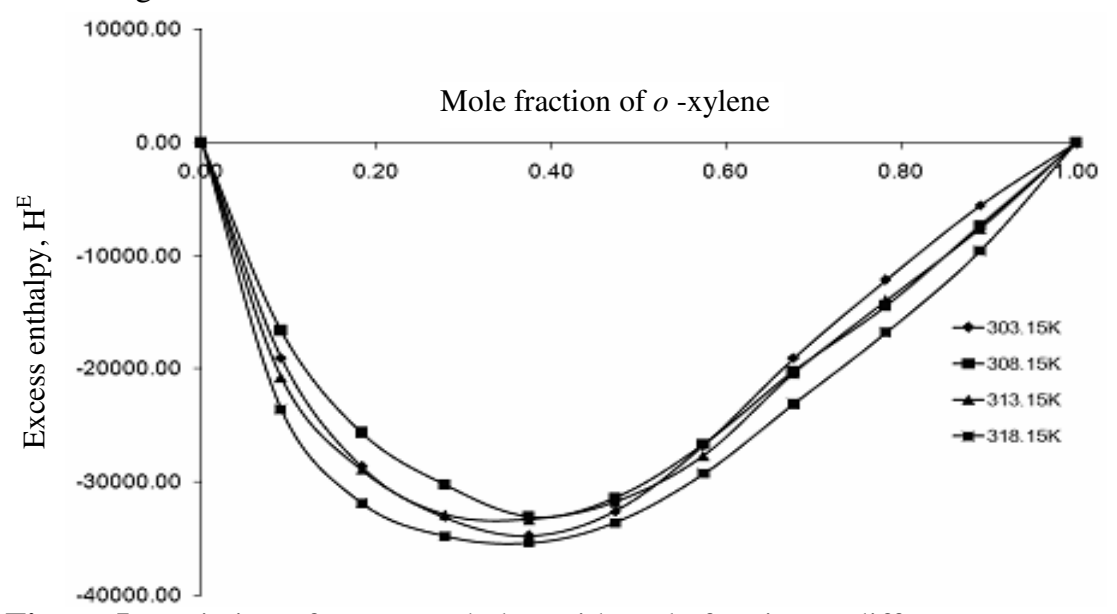

Figure 5. Variation of excess enthalpy with mole fraction at different temperatures.

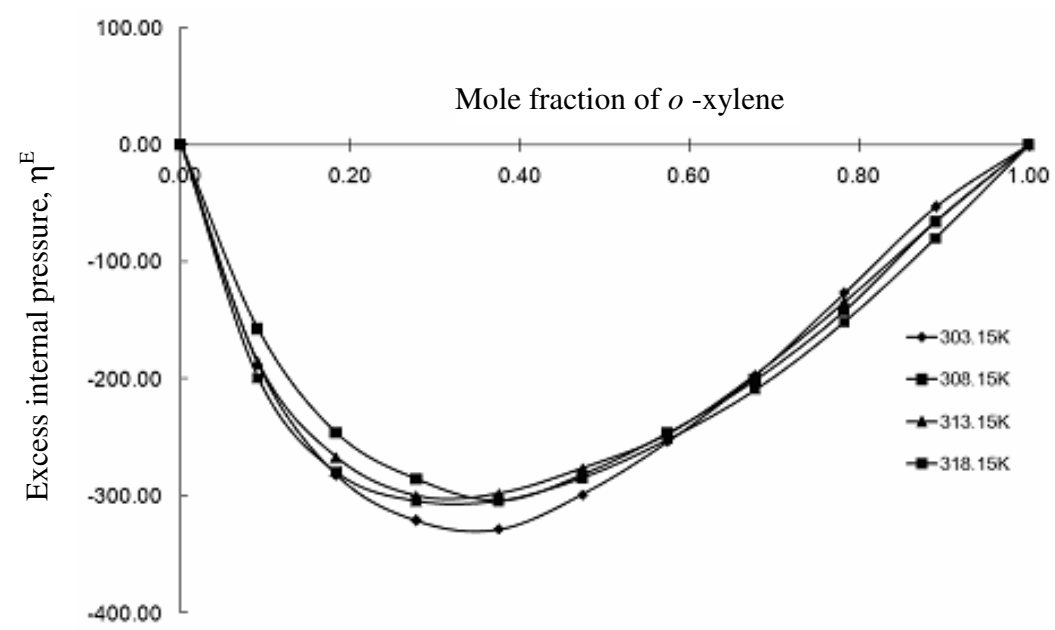

Figure 6. Variation of excess internal pressure with mole fraction at different temperatures.

The excess internal pressure is often discussed in terms of molecular interactions in liquid mixtures. The variation of excess internal pressure is entirely negative similar to the deviation in excess enthalpy and excess viscosity at all temperatures for cyclohexane with $o$-xylene. The less magnitude of these values suggests that weak interactions present in the system.

\section{Conclusion}

There are weak induced dipole-induced dipole interactions in binary mixture containing cyclohexane and $o$-xylene at temperatures $303.15,308.15,313.15$ and $318.15 \mathrm{~K}$. 


\section{References}

1. Mehra R and Israni R, Indian J Pure Appl Phys., 2000, 38, 81.

2. Oswal S I and Patel N B, J Chem Eng Data, 1995, 40, 845.

3. Anwar Ali and Nain A K, Indian J Pure Appl Phys., 2001, 39, 421.

4. Krestov G A, Thermodynamics of Solvation (Ellis Horwood, England), 1991, p.151.

5. Suryanarayanan C V and Kuppusamy J, J Acous Soc India, 1976, 4, 75.

6. Suryanarayanan C V, Indian J Chem., 1986, 25A, 538. 


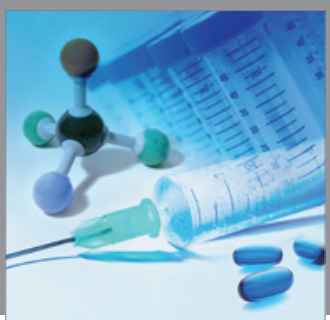

International Journal of

Medicinal Chemistry

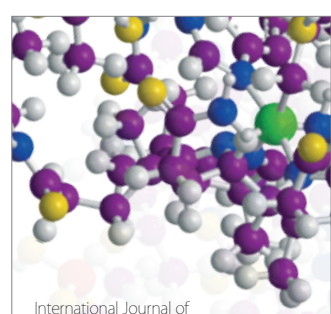

Carbohydrate Chemistry

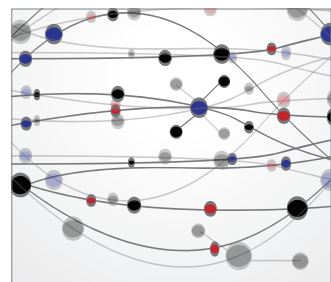

The Scientific World Journal
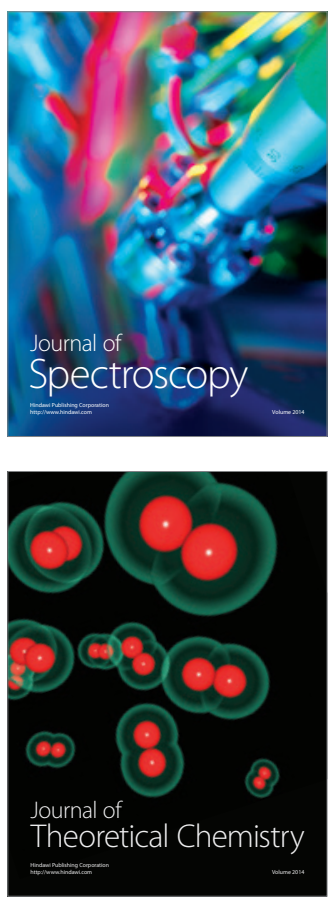
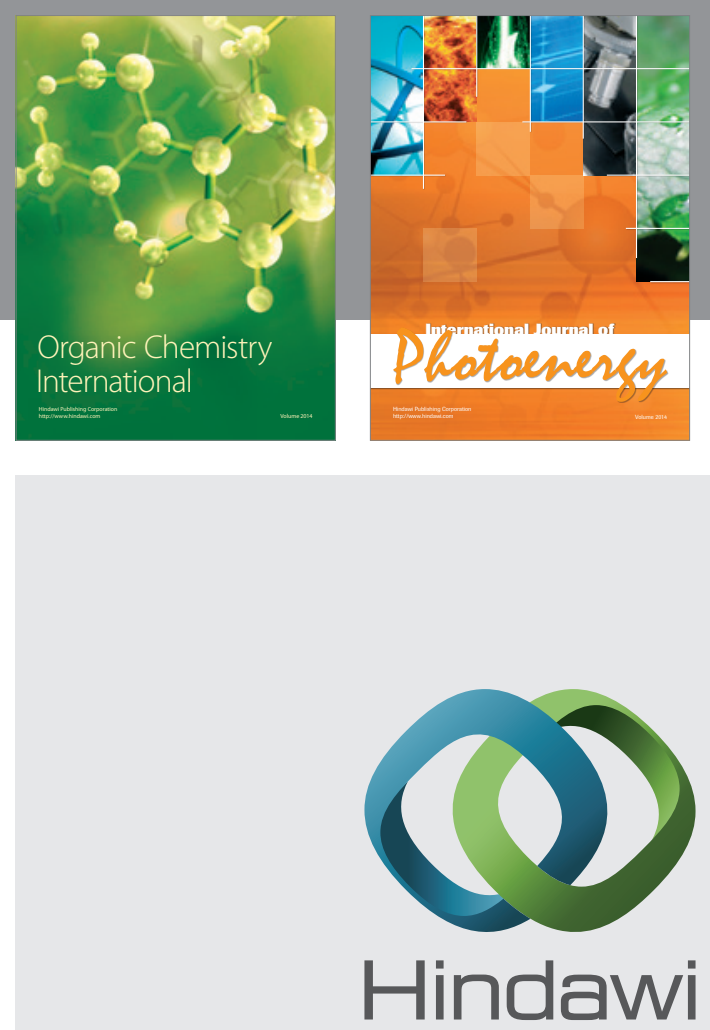

Submit your manuscripts at

http://www.hindawi.com
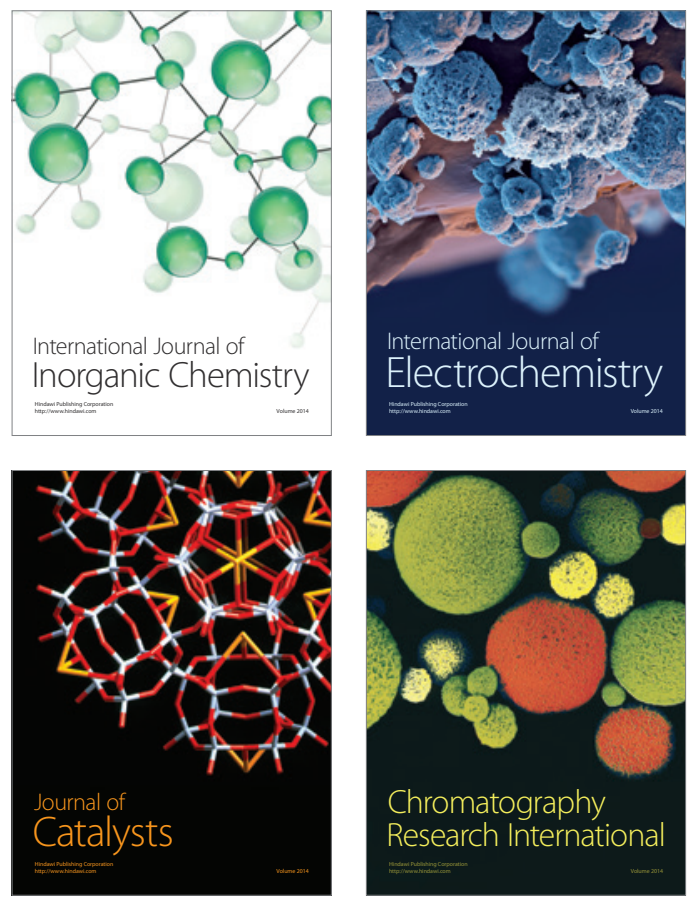
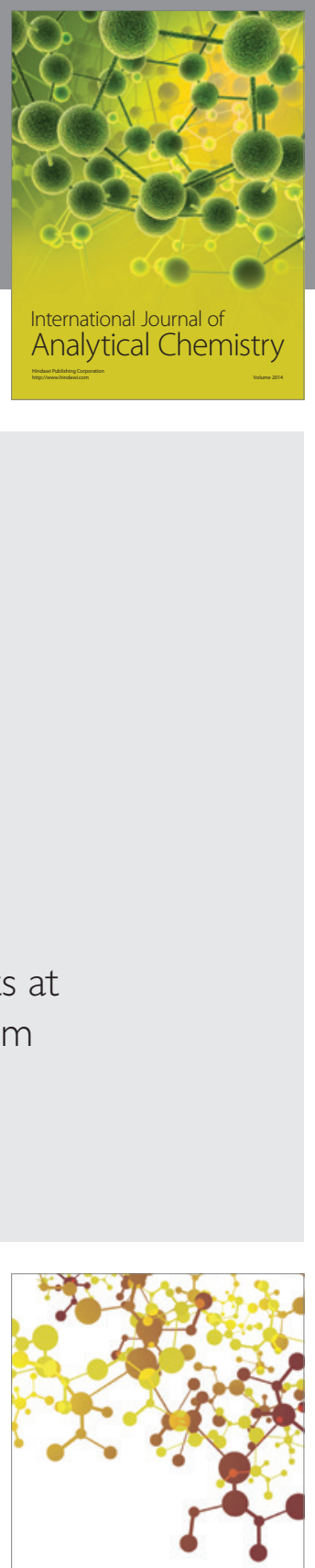

Journal of

Applied Chemistry
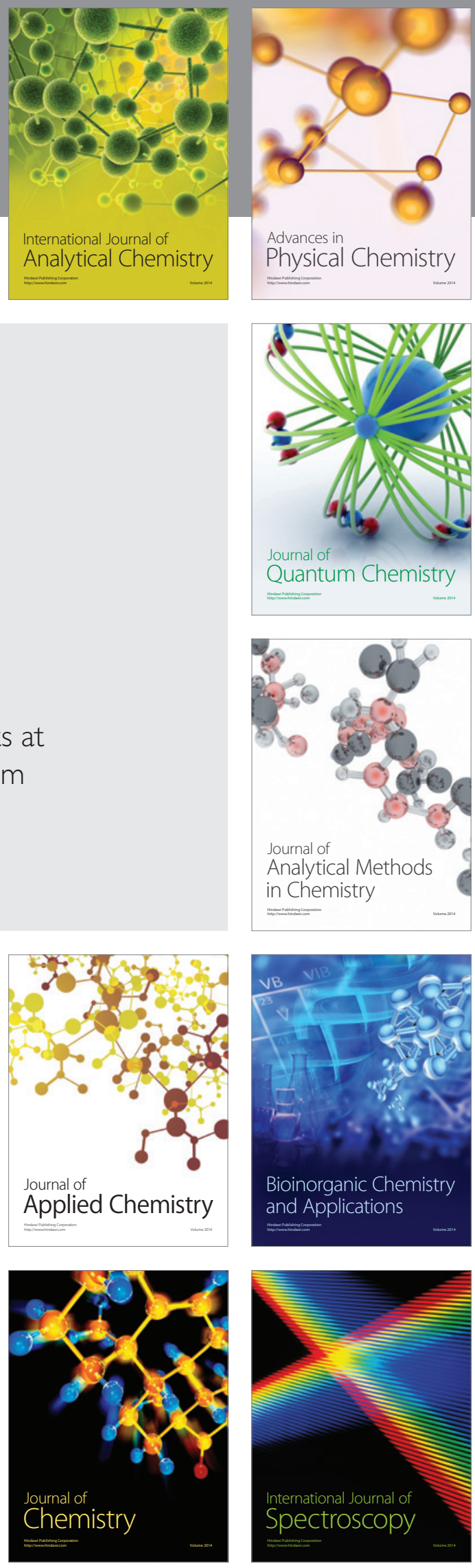